\title{
Mathematical modeling of formation and supporting of the structure of the root apical meristem Arabidopsis thaliana L.
}

\author{
V.V. Lavrekha ${ }^{1,2}$, V.V. Mironova ${ }^{1,2 *}$ \\ ${ }^{1}$ Institute of Cytology and Genetics SB RAS, Novosibirsk, Russia \\ ${ }^{2}$ Novosibirsk State University, Novosibirsk, Russia \\ *e-mail: victoria.v.mironova@gmail.com
}

Key words: mathematical modeling, auxin, cytokinin, root apical meristem, cell cycle, stem cell niche

Motivation and Aim: The stereotypic root structure, formed of concentric cell layers (the epidermis, cortex, endodermis, and pericycle, which encircle the central vascular system), is generated by the stem cell niche in the root apical meristem (RAM). The apparent simplicity of the root structure derives from an intricate pattern of developmental trajectories inside the RAM. E. g. in Arabidopsis, the diarch symmetry of vascular tissues in the root, including the pericycle, disturbs the radial root structure of the RAM. Plant hormones auxin and cytokinin play major roles in both cell division and differentiation. From another hand, their distributions are known to be nonuniform in the RAM with maxima and gradients. Here we infer the minimal regulatory mechanism for longitudinal patterning of the RAM within the gradients of auxin and cytokinin.

Methods and Algorithms: We hypothesized a plausible mechanism for regulation of the cell cycle in the RAM by auxin and cytokinin. We implemented the mechanism in 1D mathematical model. The hybrid model created using Dynamic grammar in the Plenum package for the Mathematica system. It simulates cell growth and division under control of the proposed circuit, wherein cell growth, active and passive transport of auxin and cytokinin between the cells, and their degradation are described by continuous functions. Transitions between the cell cycle phases are discrete events, which are described by stochastic rules.

Results: In the numerical calculation of the model, we observed the self-organization the proliferation domain between the maximum of auxin in the quiescent center (QC) and the maximum of cytokinin on its shootward edge. The model predicted the difference in length of proliferation activity for different vascular lineages. The model predictions have been verified experimentally.

Conclusion: Auxin and cytokinin regulate the size of the proliferation domain of RAM through concentration-dependent control of the mitotic cycle.

Acknowledgements: The work was supported by Russian Federation President Grant for young scientists (MK-1297.2017.4) and the Russian Ministry of Science and Education under 5-100 Excellence Programme. 